J. DIFFERENTIAL GEOMETRY

68 (2004) 73-98

\title{
A GEOMETRIC ANALOGUE OF THE BIRCH AND SWINNERTON-DYER CONJECTURE OVER THE COMPLEX NUMBER FIELD
}

\author{
Ken-ICHi SugIYAma
}

\begin{abstract}
We will define a Ruelle-Selberg type zeta function for a certain lomathcal system over a Riemann surface whose genus is greater than or equal to three. Also, we will investigate its property, especially their special values. As an application, we will show that a geometric analogue of BSD conjecture is true for a family of abelian varieties which has only semi-stable reductions defined over the complex number field.
\end{abstract}

\section{Introduction}

Suppose we are given an abelian variety $A$ defined over a number field $K$. Then, it is associated to an $L$-function $L_{A / K}(s)$ which absolutely converges on $\operatorname{Re} s>(3 / 2)$. It is conjectured that $L_{A / K}(s)$ can be analytically continued to an entire function throughout the $s$-plane. Moreover, the Birch and Swinnerton-Dyer conjecture predicts that its order of zero at $s=1$ is equal to the rank of the Mordell-Weil group

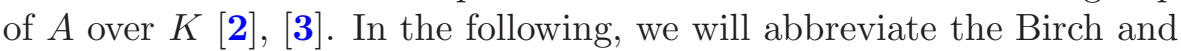
Swinnerton-Dyer conjecture to the BSD conjecture.

Artin and Tate considered a geometric analogue of the BSD conjec-

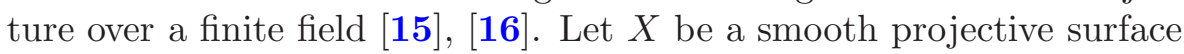
over a finite field which has an elliptic fibration $X \stackrel{f}{\rightarrow} S$ on a complete smooth curve $S$. Suppose that the moduli of $X \stackrel{f}{\rightarrow} S$ is not a constant. Using the Frobenius action on $H^{1}\left(\bar{X}_{s}, \mathbf{Q}_{l}\right)$, they associate to it an $L$-function $L_{X / S}(s)$, which is an analogue of $L_{A / K}(s)$. Here, $X_{s}$ is a special fibre at $s \in S$ and $\bar{X}_{s}$ is the base extension of $X_{s}$ to the separable closure $k(s)^{\mathrm{sep}}$ of the residue field $k(s)$. Artin and Tate conjectured that

its order of zero at $s=1$ is equal to the rank of the Mordell-Weil group of $X / S$. Moreover, Tate has shown their conjecture is equivalent to the

Received 10/10/2003. 
statement that the $l$-primary part $B r(X)(l)$ of the Brauer group of $X$ is finite [i] $\left.\mathbf{6}^{\mathbf{6}}\right]$.

We want to consider a geometric analogue of the BSD conjecture over C.

Let

$$
X \stackrel{f}{\longrightarrow} S
$$

be a commutative group scheme defined over a smooth projective curve whose generic fiber is an abelian variety of dimension $d$. Moreover, we assume the fibration satisfies all of the following conditions:

\section{Condition 1.1.}

(1) Let $\Sigma$ be a subset of $S$ where the fibration degenerates. The fibration is the Neron's model of the generic fibre which has a semi-stable reduction at each point of $\Sigma$.

(2) We set

$$
S_{0}=S \backslash \Sigma \text {. }
$$

Then the Euler-Poincaré characteristic of $S_{0}$ is negative.

(3) There is a discrete subgroup $\Gamma$ in $S L_{2}(\mathbf{R})$ such that $-1_{2} \notin \Gamma$ and $S_{0}=\Gamma \backslash \mathcal{H}$. Let us fix a base point $x_{0}$ of $S_{0}$ and we will identify $\pi_{1}\left(S_{0}, x_{0}\right)$ and $\Gamma$.

(4) We have a monodromy representation

$$
\Gamma \simeq \pi_{1}\left(S_{0}, x_{0}\right) \stackrel{\rho_{X}}{\longrightarrow} \operatorname{Aut}(V), \quad V=H^{1}\left(f^{-1}\left(x_{0}\right), \mathbf{R}\right) .
$$

Then, there is a positive constant $\alpha$ and $C$ such that

$$
\left|\operatorname{Tr} \rho_{X}(\gamma)\right| \leq C e^{\alpha l(\gamma)}
$$

is satisfied for any hyperbolic $\gamma \in \Gamma$.

(1) The moduli of the fibration is not a constant. More precisely, it satisfies

$$
H^{0}\left(S, R^{1} f_{*} \mathcal{O}_{X}\right)=0 .
$$

By the monodromy theorem [11], (1) implies $\Gamma$ has no elliptic element. The Conditions (3) and (4) are not so restrictive. For example, if necessary taking a subgroup of finite index, the Condition (3) will be always satisfied. Also, it is easy to see that the Condition (4) is satisfied if the monodromy representation is a restriction of an algebraic group homomorphism from $S L_{2}(\mathbf{R})$ to $G L_{2 d}(\mathbf{R})$ to $\Gamma$.

In order to define the Selberg and the Ruelle zeta functions of the fibration, we fix our notations.

Let $\Gamma_{\text {conj }}^{*}$ be the set of non-trivial conjugacy classes of $\Gamma$ and let $\Gamma_{h, \text { conj }}^{*}$ be its subset consisting of hyperbolic conjugacy classes. There is a natural bijection between $\Gamma_{h \text {,conj }}^{*}$ and the set of non-trivial closed geodesics 
$\pi(M)^{*}$, and we will identify them. Then, $\gamma \in \Gamma_{h, \text { conj }}^{*}$ is uniquely written as

$$
\gamma=\gamma_{0}^{\mu(\gamma)}
$$

where $\gamma_{0}$ is a primitive closed geodesic and $\mu(\gamma)$ is a positive integer, which will be called the multiplicity of $\gamma$. The subset of $\Gamma_{h \text {,conj }}^{*}$ consisting of primitive closed geodesics will be denoted by $\Gamma_{\mathrm{pr}, \mathrm{conj}}^{*}$. The length $l(\gamma)$ of $\gamma \in \Gamma_{h, \text { conj }}^{*}$ is defined to be the length of the corresponding closed geodesic. Finally, we set

$$
D(\gamma)=e^{\frac{1}{2} l(\gamma)}-e^{-\frac{1}{2} l(\gamma)} .
$$

Now the Selberg zeta function $\zeta_{S, f}(s)$ of the fibration is defined to be

$$
\zeta_{S, f}(s)=\exp \left(-\sum_{\gamma \in \Gamma_{h, \text { conj }}^{*}} \frac{2 \operatorname{Tr} \rho_{X}(\gamma)}{D(\gamma) \mu(\gamma)} e^{-s l(\gamma)}\right)
$$

and we define the Ruelle zeta function $\zeta_{R, f}(s)$ to be

$$
\zeta_{R, f}(s)=\frac{\zeta_{S, f}\left(s-\frac{1}{2}\right)}{\zeta_{S, f}\left(s+\frac{1}{2}\right)} .
$$

It is easy to see that $\zeta_{S, f}(s)$ absolutely converges on $\{s \in \mathbf{C} \mid \operatorname{Re} s>$ $(1 / 2)+\alpha\}$ and we will show it can be meromorphically continued throughout the whole plane. Also, it will be shown that $\zeta_{S, f}(s)\left(\operatorname{resp} . \zeta_{R, f}(s)\right)$ is regular at $s=0$ (resp. $s=1 / 2$ ). Our interest is $\operatorname{ord}_{s=0} \zeta_{S, f}(s)$ and $\operatorname{ord}_{s=(1 / 2)} \zeta_{R, f}(s)$.

Theorem 1.2. Let $X(S)$ be the Mordell-Weil group of the fibration. Then we have

$$
2 \operatorname{dim}_{\mathbf{Q}} X(S) \otimes \mathbf{Q} \leq \operatorname{ord}_{s=0} \zeta_{S, f}(s)=\operatorname{ord}_{s=\frac{1}{2}} \zeta_{R, f}(s) .
$$

Moreover, suppose $H^{2}\left(X, \mathcal{O}_{X}\right)=0$. Then, we have equality in the above formula.

We will show that the Ruelle zeta function has an Euler product:

$$
\zeta_{R, f}(s)=c_{0} \prod_{\gamma_{0} \in \Gamma_{\mathrm{pr}, \mathrm{conj}}^{*}}\left(\operatorname{det}\left[1_{2 d}-\rho_{X}\left(\gamma_{0}\right) e^{-s l\left(\gamma_{0}\right)}\right]\right)^{2},
$$

where $c_{0}$ is a certain constant and $1_{2 d}$ be the $2 d \times 2 d$ identity matrix. Let $f$ be a meromorphic function on a domain and let $m$ be a positive integer. We will say that $f$ has a virtual zero (resp. a virtual pole) at $a \in \mathbf{C}$ of order $m$ if its logarithmic derivative is meromorphically continued throughout the plane and has a simple pole of residue $m$ (resp. $-m$ ) at $a$. Now, Theorem 1.2 implies the following: 
Theorem 1.3. (A geometric analogue of the BSD conjecture over C) The Euler product

$$
L_{X \backslash S}(s)=\prod_{\gamma_{0} \in \Gamma_{\mathrm{pr}, \mathrm{conj}}^{*}} \operatorname{det}\left[1_{2 d}-\rho_{X}\left(\gamma_{0}\right) e^{-s l\left(\gamma_{0}\right)}\right]
$$

virtually has a zero at $s=1 / 2$ whose order is greater than or equal to the rank of the Mordell-Weil group. Moreover, if $H^{2}\left(X, \mathcal{O}_{X}\right)=0$, then they are equal.

We will show that the condition $H^{2}\left(X, \mathcal{O}_{X}\right)=0$ corresponds to the finiteness of $l$-part of the Brauer group of Artin's theorem.

The author appreciates the referee who pointed out mistakes in the first draft.

\section{A formula for the Laplacian}

To begin with, we will fix our notations. Let $G$ be $S L_{2}(\mathbf{R})$ and let $\mathbf{g}$ be its Lie algebra. We set

$$
R=\frac{1}{2}\left(\begin{array}{cc}
1 & i \\
i & -1
\end{array}\right), \quad L=\frac{1}{2}\left(\begin{array}{cc}
1 & -i \\
-i & -1
\end{array}\right), \quad H=\frac{1}{i}\left(\begin{array}{cc}
0 & 1 \\
-1 & 0
\end{array}\right)
$$

and

$$
X_{0}=\frac{1}{2} H, \quad X_{1}=\frac{1}{2 i}(R-L), \quad X_{2}=\frac{1}{2}(R+L) .
$$

They satisfy relations

$$
[H, R]=2 R, \quad[H, L]=-2 L, \quad[R, L]=H
$$

and

$$
\left[X_{1}, X_{2}\right]=\frac{1}{i} X_{0}, \quad X_{1}^{2}+X_{2}^{2}=\frac{1}{2}(R L+L R) .
$$

Note that $\left\{i H, X_{1}, X_{2}\right\}$ forms an orthogonal basis of $\mathbf{g}$ with respect to the Killing form. For reals $x, \theta$ and for a positive $y$, we set

$n(x)=\left(\begin{array}{ll}1 & x \\ 0 & 1\end{array}\right), \quad k(\theta)=\left(\begin{array}{cc}\cos \theta & \sin \theta \\ -\sin \theta & \cos \theta\end{array}\right), \quad a(y)=\left(\begin{array}{cc}\sqrt{y} & 0 \\ 0 & \sqrt{y}^{-1}\end{array}\right)$.

According to the Iwasawa decomposition $G=N A K$, any element $g$ of $G$ is uniquely written as

$$
g=n(x(g)) a(y(g)) k(\theta(g)),
$$

where $x(g) \in \mathbf{R}, y(g)>0$, and $\theta(g) \in[0,2 \pi)$. We consider $G$ endowed with a coordinate by this parametrization and we normalize our Haar measure $d g$ of $G$ to be

$$
d g=\frac{d x d y}{y^{2}} d \theta
$$




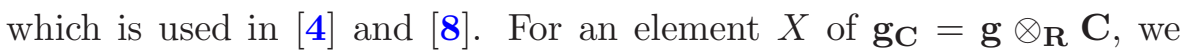
define its (right) Lie derivation $r(X)$ on $C^{\infty}(G)$ by

$$
(r(X) f)(g)=\left.\frac{d}{d t} f(g \exp (t X))\right|_{t=0}, \quad f \in C^{\infty}(G) .
$$

For example, we have

$$
r\left(X_{0}\right)=\frac{1}{2 i} \frac{d}{d \theta} .
$$

Let $\Omega$ be the Casimir element of $\mathbf{g}$ :

$$
\Omega=\frac{1}{4}\left(H^{2}+2 R L+2 L R\right)=X_{0}^{2}+X_{1}^{2}+X_{2}^{2} .
$$

It is known that the center of the universal enveloping algebra $u\left(\mathbf{g}_{\mathbf{C}}\right)$ of $\mathbf{g}_{\mathbf{C}}$ is generated by $\Omega$. Let $\mathbf{k}$ be the Lie subalgebra of $\mathbf{g}$ corresponding to the maximal compact subgroup $K(=U(1))$ of $G$ and let

$$
\mathbf{g}=\mathbf{k} \oplus \mathbf{p}
$$

be the Cartan decomposition. Then, $\mathbf{p}$ is identified with the tangent plane of the Poincaré upper half plane $\mathcal{H}$ at $i$ and $\left\{X_{1}, X_{2}\right\}$ forms an orthogonal basis of $\mathbf{p}$. Here, we always consider $\mathcal{H}$ is given the metric of the constant curvature $\equiv-1$. Let $\left\{\omega_{1}, \omega_{2}\right\}$ be its dual basis.

For an integer $m$, let $\mathbf{C}(m)$ be a unitary representation of the maximal compact subgroup $K=U(1)$ of $G$ whose action is given by

$$
\rho_{m}(k(\theta)) \mathbf{1}_{m}=e^{i m \theta} \mathbf{1}_{m},
$$

where $\mathbf{1}_{m}$ is a base of $\mathbf{C}(m)$. Note that $K$ acts on $\mathbf{p}_{\mathbf{C}}=\mathbf{p} \otimes \mathbf{C}$ and its dual space $\mathbf{p}_{\mathbf{C}}^{*}$ by the adjoint action and they have an irreducible decomposition as a $K$-module,

$$
\mathbf{p}_{\mathbf{C}} \simeq \mathbf{p}_{\mathbf{C}}^{*} \simeq \mathbf{C}(-2) \oplus \mathbf{C}(2) .
$$

Note that $\rho_{m}$ induces a homogeneous complex line bundle $\mathcal{L}_{m}$ over $\mathcal{H}$,

$$
\mathcal{L}_{m}=G \times{ }_{\left(K, \rho_{m}\right)} \mathbf{C}(m) .
$$

Then, the space of smooth sections of $\mathcal{L}_{m}\left(\right.$ resp. $\mathcal{L}_{m} \otimes_{\mathbf{C}}\left(T^{*} \mathcal{H}\right)_{\mathbf{C}}, \mathcal{L}_{m} \otimes_{\mathbf{C}}$ $\wedge^{2}\left(T^{*} \mathcal{H}\right)_{\mathbf{C}}$, where $\cdot \mathbf{C}$ denotes the complexification) is naturally identified with the $K$-invariant part $\left(C^{\infty}(G) \otimes \mathbf{C}(m)\right)^{K}$ of $C^{\infty}(G) \otimes \mathbf{C}(m)$ (resp. $\left.\left(C^{\infty}(G) \otimes \mathbf{p}_{\mathbf{C}}^{*} \otimes \mathbf{C}(m)\right)^{K},\left(C^{\infty}(G) \otimes \wedge^{2} \mathbf{p}_{\mathbf{C}}^{*} \otimes \mathbf{C}(m)\right)^{K}\right)$. By this identification, the negative Hodge Laplacian

$$
\Delta=-(d \delta+\delta d)
$$

acts on the latter spaces, where $\delta$ is the formal adjoint of $d$. In order to write down this action, we need some notations.

By definition, we have

$$
R=X_{2}+i X_{1}, \quad L=X_{2}-i X_{1} .
$$


Hence, $\left\{R_{0}=\frac{1}{\sqrt{2}} R, L_{0}=\frac{1}{\sqrt{2}} L\right\}$ forms a unitary basis of $\mathbf{g}_{\mathbf{C}}$. Note that they satisfy

$$
X_{1}^{2}+X_{2}^{2}=R_{0} L_{0}+L_{0} R_{0} .
$$

Let $\left\{\xi_{1}, \xi_{2}\right\}$ be its dual basis. According to (1), we have

$$
\operatorname{Ad}(k(\theta)) \xi_{1}=e^{-2 i \theta}, \quad \operatorname{Ad}(k(\theta)) \xi_{2}=e^{2 i \theta} .
$$

Hence, (2) is nothing but the obvious identity

$$
\mathbf{p}_{\mathbf{C}}^{*}=\mathbf{C} \xi_{1} \oplus \mathbf{C} \xi_{2} .
$$

Now, taking account of identities

$$
X_{1} \cdot \mathbf{1}_{m}=X_{2} \cdot \mathbf{1}_{m}=0,
$$

the following proposition will be obtained by a simple computation.

Proposition 2.1. Let $F_{1}, F_{2}$ and $f$ be smooth functions on $G$. Suppose each $\alpha=f \otimes \mathbf{1}_{m}, \beta=F_{1} \otimes \mathbf{1}_{m} \otimes \xi_{1}+F_{2} \otimes \mathbf{1}_{m} \otimes \xi_{2}$ and $\gamma=$ $f \otimes \mathbf{1}_{m} \otimes\left(\xi_{1} \wedge \xi_{2}\right)$ is $K$-invariant. Then, we have

(1)

$$
\begin{gathered}
\Delta(\alpha)=\left(r(\Omega)-\frac{m^{2}}{4}\right) f \otimes \mathbf{1}_{m} . \\
\Delta(\beta)=\left(r(\Omega)-\left(1-\frac{m}{2}\right)^{2}+\left(1-\frac{m}{2}\right)\right) F_{1} \otimes \mathbf{1}_{m} \otimes \xi_{1} \\
+\left(r(\Omega)-\left(1+\frac{m}{2}\right)^{2}+\left(1+\frac{m}{2}\right)\right) F_{2} \otimes \mathbf{1}_{m} \otimes \xi_{2} .
\end{gathered}
$$

$(3)$

$$
\Delta(\gamma)=\left(r(\Omega)-\frac{m^{2}}{4}\right) f \otimes \mathbf{1}_{m} \otimes\left(\xi_{1} \wedge \xi_{2}\right) .
$$

Example 2.2. Let $\alpha, \beta$, and $\gamma$ be the same as above. Suppose that $m= \pm 1$. Then, we have

$$
\Delta(\alpha)=\left(r(\Omega)-\frac{1}{4}\right) f \otimes \mathbf{1}_{m}, \quad m= \pm 1 .
$$

$$
\Delta(\beta)=\left(r(\Omega)+\frac{1}{4}\right) F_{1} \otimes \mathbf{1}_{m} \otimes \xi_{1}+\left(r(\Omega)-\frac{3}{4}\right) F_{2} \otimes \mathbf{1}_{m} \otimes \xi_{2}, \quad m=1 .
$$


(b)

$\Delta(\beta)=\left(r(\Omega)-\frac{3}{4}\right) F_{1} \otimes \mathbf{1}_{m} \otimes \xi_{1}+\left(r(\Omega)+\frac{1}{4}\right) F_{2} \otimes \mathbf{1}_{m} \otimes \xi_{2}, \quad m=-1$.

$$
\Delta(\gamma)=\left(r(\Omega)-\frac{1}{4}\right) f \otimes \mathbf{1}_{m} \otimes\left(\xi_{1} \wedge \xi_{2}\right), \quad m= \pm 1
$$

\section{Hodge decomposition} of locally homogeneous vector bundles

Let $\Gamma$ be a discrete subgroup of $G$ such that the volume of $M=\Gamma \backslash \mathcal{H}$ is finite and that the Euler-Poincaré characteristic $\chi(M)$ is negative. We always assume that $\Gamma$ has no elliptic element and $-1_{2} \notin \Gamma$. It is known that every hyperbolic element $\gamma \in G$ is conjugate to an element of a form

$$
\left(\begin{array}{cc}
-1 & 0 \\
0 & -1
\end{array}\right)^{m(\gamma)}\left(\begin{array}{cc}
\sqrt{y_{\gamma}} & 0 \\
0 & \sqrt{y_{\gamma}}-1
\end{array}\right)
$$

in $G$, where $m(\gamma) \in\{0,1\}$ and $y_{\gamma}$ is a positive. We will determine $m(\gamma)$

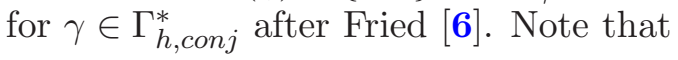

$$
\left(\begin{array}{cc}
-1 & 0 \\
0 & -1
\end{array}\right)^{m(\gamma)}
$$

is the holonomy when one parallel transports a normal vector around the closed geodesic corresponding to $\gamma$. But since $M$ is oriented, $m(\gamma)$ should be equal to 0 .

Let $V$ be a $2 d$ dimensional vector space over $\mathbf{R}$ such that $V_{\mathbf{C}}=V \otimes_{\mathbf{R}} \mathbf{C}$ has a decomposition as a $K$-module

$$
V_{\mathbf{C}}=V_{\mathbf{C}}^{1,0} \oplus V_{\mathbf{C}}^{0,1}, \quad V_{\mathbf{C}}^{1,0}=\mathbf{C}(1)^{\oplus d}, \quad V_{\mathbf{C}}^{0,1}=\mathbf{C}(-1)^{\oplus d} .
$$

Suppose $V$ has a $\Gamma$-action $\rho$. Then, the complexification $\mathcal{V}_{\mathbf{C}}$ of the vector bundle

$$
\mathcal{V}=\mathcal{H} \times_{(\Gamma, \rho)} V
$$

on $M$ and its tensor product with the cotangent bundle $\left(T^{*} M \otimes \mathcal{V}\right)_{\mathbf{C}}$ or the vector bundle of two forms $\left(\wedge^{2} T^{*} M \otimes \mathcal{V}\right)_{\mathbf{C}}$ have a Hodge decomposition in Zucker's sense, which we will recall [20].

Since the cotangent bundle $T^{*} \mathcal{H}$ of $\mathcal{H}$ is equal to $G \times{ }_{(K, \mathrm{Ad})} \mathbf{p}^{*}$, its complexification has a decomposition

$$
\left(T^{*} \mathcal{H}\right)_{\mathbf{C}}=G \times_{K} \mathbf{p}_{\mathbf{C}}^{1,0} \oplus G \times_{K} \mathbf{p}_{\mathbf{C}}^{0,1},
$$

by (2), where $\mathbf{p}_{\mathbf{C}}^{1,0}=\mathbf{C}(2)$ and $\mathbf{p}_{\mathbf{C}}^{0,1}=\mathbf{C}(-2) . G \times_{K} \mathbf{p}_{\mathbf{C}}^{1,0}$ (resp. $G \times_{K}$ $\left.\mathbf{p}_{\mathbf{C}}^{0,1}\right)$ will be denoted by $\left(T^{*} \mathcal{H}\right)_{\mathbf{C}}^{1,0}\left(\operatorname{resp} .\left(T^{*} \mathcal{H}\right)_{\mathbf{C}}^{0,1}\right)$ and they are pushed 
down to subbundles $\left(T^{*} M\right)_{\mathbf{C}}^{1.0}$ and $\left(T^{*} M\right)_{\mathbf{C}}^{0.1}$ of $\left(T^{*} M\right)_{\mathbf{C}}$ respectively. On the other hand, a homogeneous vector bundle on $\mathcal{H}$

$$
\tilde{\mathcal{V}}_{\mathbf{C}}=G \times_{K} V_{\mathbf{C}}
$$

descends to $\mathcal{V}_{\mathbf{C}}$ and the image of

$$
\tilde{\mathcal{V}}_{\mathbf{C}}^{1.0}=G \times_{K} V_{\mathbf{C}}^{1.0}, \quad \tilde{\mathcal{V}}_{\mathbf{C}}^{0.1}=G \times_{K} V_{\mathbf{C}}^{0.1}
$$

will be denoted by $\mathcal{V}_{\mathbf{C}}^{1.0}$ and $\mathcal{V}_{\mathbf{C}}^{0.1}$, respectively. Now, the $(p, q)$-part $\left(T^{*} M \otimes \mathcal{V}\right)_{\mathbf{C}}^{p . q}$ of $\left(T^{*} M \otimes \mathcal{V}\right)_{\mathbf{C}}$ is defined to be

$$
\left(T^{*} M \otimes \mathcal{V}\right)_{\mathbf{C}}^{p . q}=\oplus_{a+c=p, b+d=q}\left(T^{*} M\right)_{\mathbf{C}}^{a . b} \otimes \mathcal{V}_{\mathbf{C}}^{c . d} .
$$

Note that this is nothing but the descent of

$$
\tilde{\mathcal{W}}^{p \cdot q}=G \times{ }_{K} W^{p . q}, \quad W^{p \cdot q}=\oplus_{a+c=p, b+d=q}\left(\mathbf{p}_{\mathbf{C}}^{a . b} \otimes V_{\mathbf{C}}^{c . d}\right) .
$$

Since $d$-copies of $\left\{\mathbf{1}_{1} \otimes \xi_{1}, \mathbf{1}_{-1} \otimes \xi_{2}\right\}$ (resp. $\left\{\mathbf{1}_{1} \otimes \xi_{2}, \mathbf{1}_{-1} \otimes \xi_{1}\right\}$ ) forms a basis of $W^{1.1}$ (resp. $W^{2.0} \oplus W^{0.2}$ ), Example 2.21 implies the following formulae.

Proposition 3.1. Let $f \otimes w \in\left(C^{\infty} \otimes W^{p \cdot q}\right)^{K}$.

(1) If $(p . q)=(1.1)$,

$$
\Delta(f \otimes w)=\left(\left(r(\Omega)+\frac{1}{4}\right) f\right) \otimes w .
$$

(2) If $(p . q)=(2.0)$, or $(0.2)$,

$$
\Delta(f \otimes w)=\left(\left(r(\Omega)-\frac{3}{4}\right) f\right) \otimes w .
$$

Proposition 3.2. (1) If $f \otimes v \in\left(C^{\infty} \otimes V_{\mathbf{C}}\right)^{K}$,

$$
\Delta(f \otimes v)=\left(\left(r(\Omega)-\frac{1}{4}\right) f\right) \otimes v .
$$

(2) If $f \otimes v \in\left(C^{\infty} \otimes V_{\mathbf{C}} \otimes \wedge^{2} T^{*}(M)\right)^{K}$,

$$
\Delta\left(f \otimes v \otimes\left(\xi_{1} \wedge \xi_{1}\right)\right)=\left(\left(r(\Omega)-\frac{1}{4}\right) f\right) \otimes v \otimes\left(\xi_{1} \wedge \xi_{1}\right) .
$$

\section{The heat kernel of the positive Hodge Laplacian}

In the sequel to the paper, we shall only treat the vector bundle $\left(T^{*} M \otimes \mathcal{V}\right)_{\mathbf{C}}^{1.1}$. Let $\mu$ be the action of $K$ on $W^{1.1} \simeq(\mathbf{C}(1) \oplus \mathbf{C}(-1))^{\oplus d}$. As we have seen in the previous section, this is the descent of the homogeneous vector bundle

$$
\tilde{\mathcal{W}}^{1.1}=G \times{ }_{(K, \mu)} W^{1.1}
$$


on $\mathcal{H}$ to $M$. After Barbasch and Moscovici [1] ], we will regard $C^{\infty}(G) \otimes$ $\operatorname{End}\left(W^{1.1}\right)$ as $K \times K$-module via the action

$$
\left(\rho\left(k_{1}, k_{2}\right) f \otimes A\right)(g)=f\left(k_{1} g k_{2}\right) \otimes \mu\left(k_{1}\right)^{-1} A \mu\left(k_{2}\right)^{-1},
$$

where $k_{i}$ (resp. $g$ ) is an element of $K\left(\right.$ resp. $G$ ) and $f \otimes A \in C^{\infty}(G) \otimes$ $\operatorname{End}\left(W^{1.1}\right)$. So, $h \in C^{\infty}(G) \otimes \operatorname{End}\left(W^{1.1}\right)$ is $K \times K$-invariant if and only if it satisfies the covariance property

$$
h\left(k_{1} g k_{2}\right)=\mu\left(k_{1}\right) h(g) \mu\left(k_{2}\right) .
$$

Let $\Delta_{H}=-\Delta$ be the positive Hodge Laplacian and let $\Delta_{G}$ be the Laplacian of $G$

$$
\Delta_{G}=-r(\Omega)+2 r\left(X_{0}\right)^{2} .
$$

It is known that the heat kernel $p_{t}$ of $G$ is contained in $C^{\infty}(G) \cap L^{1} \cap L^{2}$ and that it satisfies

$$
\left(e^{-t \Delta_{G}} u\right)(x)=\int_{G} p_{t}\left(x^{-1} y\right) u(y) d y, \quad u \in L^{2}(G)
$$

for any $t>0$ [i] be the right regular representation of $G$ and we set

$$
Q_{R}=\int_{K} R(k) \otimes \mu(k) d k .
$$

Then, $Q_{R}$ is a projection from $C^{\infty}(G) \otimes W^{1.1}$ to its $K$-invariant part

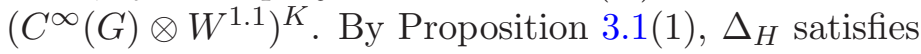

$$
\Delta_{H}=Q_{R} \circ\left(\Delta_{G} \otimes \operatorname{Id}_{W^{1.1}}\right) \circ Q_{R}-\frac{3}{4},
$$

and the kernel function $h_{t}^{H}(t>0)$ of $e^{-t \Delta_{H}}$ is given by

$$
h_{t}^{H}(x)=e^{\frac{3}{4} t} \int_{K} d k_{1} \mu\left(k_{1}\right)^{-1} \int_{K} p_{t}\left(k_{1} x k_{2}\right) \mu\left(k_{2}\right)^{-1} d k_{2} .
$$

Note that $h_{t}^{H}$ is contained in $\left[\left(C^{\infty}(G) \cap L^{1} \cap L^{2}\right) \otimes \operatorname{End}\left(\mathrm{W}^{1.1}\right)\right]^{K \times K}$ and since $K$ is commutative, it satisfies

$$
\mu(k) h_{t}^{H}(x)=h_{t}^{H}(x) \mu(k), \quad x \in G, k \in K .
$$

The following lemma is an immediate consequence of Proposition

Lemma 4.1. Let $\pi$ be an irreducible unitary representation of $G$ and let $\mathcal{H}(\pi)$ be its representation space. Then

$$
\pi\left(h_{t}^{H}\right)=e^{t\left(\pi(\Omega)+\frac{1}{4}\right)} \mathrm{Id}
$$

on $\left(\mathcal{H}(\pi) \otimes W^{1.1}\right)^{K}$. 
Let $\pi$ be an irreducible unitary representation of $G$. By the Frobenius

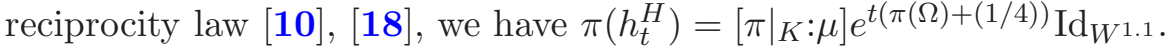

For example, let us take $\pi$ to be the principal series $\pi_{\nu, 1}$ of a parameter $(s, \epsilon)=((1 / 2)+i \nu, 1)[4]$. Using the explicit description of $\pi_{\nu, 1}$, one obtains $\left[\left.\pi_{\nu, 1}\right|_{K}: \mu\right]=1$ and $\pi_{\nu, 1}(\Omega)=-\left((1 / 4)+\nu^{2}\right)$. Therefore, we have

$$
\pi_{\nu, 1}\left(h_{t}^{H}\right)=e^{-t \nu^{2}} \operatorname{Id}_{W^{1.1}} \text {. }
$$

For $p>0$, let $\mathcal{C}^{p}(G)$ be the Harish-Chandra's $\mathcal{C}^{p}$-space of $G$. Since it is known $h_{t}^{H}(t>0)$ is contained in $\mathcal{C}^{p}(G) \otimes \operatorname{End}\left(W^{1.1}\right)$ [1], [1] ne may apply it the Selberg trace formula. This will be the main object of the following sections.

\section{The Selberg trace formula}

For brevity, we set $\mathcal{W}^{p \cdot q}=\left(T^{*} M \otimes \mathcal{V}\right)_{\mathbf{C}}^{p . q}$ and let $L^{2}\left(M, \mathcal{W}^{p . q}\right)$ be the space of square integrable sections with respect to the Poincaré metric. We will only treat the case of $(p \cdot q)=(1.1)$.

There is an orthogonal decomposition

$$
L^{2}\left(M, \mathcal{W}^{1.1}\right)=L^{2}\left(M, \mathcal{W}^{1.1}\right)_{\operatorname{disc}} \oplus L^{2}\left(\mathcal{W}^{1.1}\right)_{\mathrm{cont}},
$$

according to the type of spectra of $\Delta_{H}$. The trace

$$
\operatorname{Tr}\left[e^{-t \Delta_{H}} \mid L^{2}\left(M, \mathcal{W}^{1.1}\right) \text { disc }\right]
$$

can be computed by the Selberg trace formula and consists of three

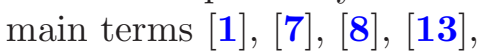

$$
\operatorname{Tr}\left[e^{-t \Delta_{H}} \mid L^{2}\left(M, \mathcal{W}^{1.1}\right)_{\text {disc }}\right]=I(t)+G(t)+P(t) .
$$

$I(t)$ (resp. $G(t))$ is the orbital integral over $1_{2}$ (resp. hyperbolic orbits). $P(t)$ is the orbital integral on parabolic orbits minus a contribution of continuous spectra. Also, $G(t)$ will be concerned with the Ruelle and Selberg zeta functions. Each term will be explicitly computed in the later sections.

If 0 is a spectrum of $\Delta_{H}$, it is known that it is a discrete one and $\operatorname{ker}\left[\Delta_{H} \mid L^{2}\left(M, \mathcal{W}^{1.1}\right)\right]$ is a subspace of $L^{2}\left(M, \mathcal{W}^{1.1}\right)_{\operatorname{disc}}\left[\mathbf{1} \mathbf{1}_{-1}\right]\left[\mathbf{2}_{-}^{\mathbf{2}} \mathbf{0}\right]$. We set

$$
b=\operatorname{dim}_{\mathbf{C}} \operatorname{ker}\left[\Delta_{H} \mid L^{2}\left(M, \mathcal{W}^{1.1}\right)\right]
$$

and let

$$
0=\lambda_{1}=\cdots=\lambda_{b}<\lambda_{b+1} \leq \lambda_{b+2} \leq \cdots,
$$

be the discrete spectra of $\Delta_{H}$. The following proposition is a consequence of an easy computation.

Proposition 5.1. The integral

$$
2 s \int_{0}^{\infty} e^{-s^{2} t} \operatorname{Tr}\left[e^{-t \Delta_{H}} \mid L^{2}\left(M, \mathcal{W}^{1.1}\right)_{\text {disc }}\right] d t
$$


exists for $-\frac{\pi}{4}<\arg s<\frac{\pi}{4}$ and it can be meromorphically continued throughout the s-plane as

$$
\frac{2 b}{s}+\sum_{n=b+1}^{\infty}\left(\frac{1}{s+\sqrt{\lambda_{n}} i}+\frac{1}{s-\sqrt{\lambda_{n}} i}\right) .
$$

\section{Ruelle and Selberg zeta functions}

Taking account of $m(\gamma)=0$ for $\gamma \in \Gamma_{h, \text { conj }}^{*}$ (cf. Section $\left.\underline{\underline{\beta}}_{\mathbf{1}}\right)$ ) and using the identity (6), the orbital integral $G(t)$ of $h_{t}^{H}$ on hyperbolic orbits (cf.

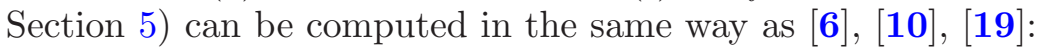

$$
G(t)=\sum_{\gamma \in \Gamma_{h, \text { conj }}^{*}} \frac{l\left(\gamma_{0}\right) \operatorname{Tr} \rho(\gamma)}{\pi D(\gamma)} \int_{-\infty}^{\infty} e^{-t \nu^{2}-i l(\gamma) \nu} d \nu .
$$

(Also, we have used the fact that the centralizer of

$$
A=\left\{\left(\begin{array}{cc}
a & 0 \\
0 & a^{-1}
\end{array}\right) \mid a>0\right\}
$$

in $K$ is $\{ \pm 1\}$.) Here, $D(\gamma)$ is defined to be

$$
D(\gamma)=e^{\frac{1}{2} l(\gamma)}-e^{-\frac{1}{2} l(\gamma)} .
$$

Inserting the formula

$$
\int_{-\infty}^{\infty} e^{-t \nu^{2}-i l(\gamma) \nu} d \nu=\sqrt{\frac{\pi}{t}} e^{-\frac{l(\gamma)^{2}}{4 t}}
$$

to the above identity, we get the following proposition.

Proposition 6.1.

$$
G(t)=\sum_{\gamma \in \Gamma_{h, \text { conj }}^{*}} \frac{l\left(\gamma_{0}\right) \operatorname{Tr} \rho(\gamma)}{\sqrt{\pi t} D(\gamma)} e^{-\frac{l(\gamma)^{2}}{4 t}}, \quad t>0 .
$$

Now, we define our Selberg zeta function.

Definition 6.2. The Selberg zeta function $\zeta_{S}(s)$ is defined to be

$$
\zeta_{S}(s)=\exp \left(-\sum_{\gamma \in \Gamma_{h, \text { conj }}^{*}} \frac{2 \operatorname{Tr} \rho(\gamma)}{D(\gamma) \mu(\gamma)} e^{-s l(\gamma)}\right) .
$$

Let $Z_{S}(s)$ be its logarithmic derivative,

$$
Z_{S}(s)=\frac{\zeta_{S}^{\prime}(s)}{\zeta_{S}(s)} .
$$

For the sake of convergence of the Selberg zeta function, we always assume $\rho$ satisfies the following technical condition. 
Condition 6.3. There exists positive constants $C$ and $\alpha$ such that

$$
|\operatorname{Tr} \rho(\gamma)| \leq C e^{\alpha l(\gamma)}
$$

is satisfied for any hyperbolic $\gamma \in \Gamma$.

Under the condition, it is easy to see $\zeta_{S}(s)$ absolutely converges for $\operatorname{Re} s>\alpha+(1 / 2)[\overline{9} \mathbf{j}]$. Note that $Z_{S}(s)$ has a form

$$
Z_{S}(s)=\sum_{\gamma \in \Gamma_{h, \text { conj }}^{*}} \frac{2 l\left(\gamma_{0}\right) \operatorname{Tr} \rho(\gamma)}{D(\gamma)} e^{-s l(\gamma)} .
$$

There is an intimate relation between $G(t)$ and $Z_{S}(s)$.

Proposition 6.4. For $s \in \mathbf{C}$ with $-\frac{\pi}{4}<\arg s<\frac{\pi}{4}$ and $\operatorname{Re} s$ is sufficiently large, we have the identity

$$
2 s \int_{0}^{\infty} e^{-s^{2} t} G(t) d t=Z_{S}(s)
$$

Proof. It is easy to see the integral converges for such $s$. Since both sides are analytic functions with respect to $s$, it is sufficient to check the identity for $s>0$. Applying a change of variables

$$
x=s \sqrt{t}
$$

to the formula

$$
\int_{0}^{\infty} e^{-\left(x^{2}+\frac{a^{2}}{x^{2}}\right)} d x=\frac{\sqrt{\pi}}{2} e^{2 a}, \quad \operatorname{Re} a^{2}>0
$$

we get

$$
\frac{1}{2} e^{-s l(\gamma)}=s \int_{0}^{\infty} \frac{1}{\sqrt{4 \pi t}} e^{-s^{2} t-\frac{l(\gamma)^{2}}{4 t}} d t
$$

Combining this with Proposition 6.1, the assertion will be proved. q.e.d.

Definition 6.5. The Ruelle zeta function $\zeta_{R}(s)$ is defined to be

$$
\zeta_{R}(s)=\frac{\zeta_{S}\left(s-\frac{1}{2}\right)}{\zeta_{S}\left(s+\frac{1}{2}\right)} .
$$

In the later sections, we will show that both Selberg and Ruelle zeta functions can be meromorphically continued throughout the $s$-plane. Also, $\zeta_{R}(s)$ has an Euler product.

Proposition 6.6. We have an identity

$$
\zeta_{R}(s)=c_{0} \prod_{\gamma_{0} \in \Gamma_{\mathrm{pr}, \mathrm{conj}}^{*}}\left(\operatorname{det}\left[1_{2 d}-\rho\left(\gamma_{0}\right) e^{-s l\left(\gamma_{0}\right)}\right]\right)^{2} .
$$


Proof. We will compare both sides taking their logarithmic derivatives. The logarithmic derivative of $\zeta_{R}(s)$ is

$$
\begin{aligned}
\frac{\zeta_{R}^{\prime}(s)}{\zeta_{R}(s)} & =\sum_{\gamma \in \Gamma_{h, \mathrm{conj}}^{*}} \frac{2 l\left(\gamma_{0}\right) \operatorname{Tr} \rho(\gamma)}{D(\gamma)}\left(e^{-\left(s-\frac{1}{2}\right) l(\gamma)}-e^{-\left(s+\frac{1}{2}\right) l(\gamma)}\right) \\
& =2 \sum_{\gamma \in \Gamma_{h, \mathrm{conj}}^{*}} l\left(\gamma_{0}\right) \operatorname{Tr} \rho(\gamma) e^{-s l(\gamma)} \\
& =2 \sum_{\gamma \in \Gamma_{\mathrm{pr}, \mathrm{conj}}^{*}} l\left(\gamma_{0}\right) \sum_{k=1}^{\infty} \operatorname{Tr} \rho\left(\gamma_{0}^{k}\right) e^{-s k l\left(\gamma_{0}\right)} .
\end{aligned}
$$

Note that the last equation is just the logarithmic derivative of the RHS of the required identity.

q.e.d.

\section{Orbital integrals}

First of all, we will fix our notations.

According to the Iwasawa decomposition

$$
G=N A K
$$

every element $g$ of $G$ may be uniquely written to be

$$
g=n(g) a(g) k(g), \quad n(g) \in N, \quad a(g) \in A, \quad k(g) \in K .
$$

We give a coordinate on $A$ by

$$
\mathbf{R}_{>0}=\{x \in \mathbf{R} \mid x>0\} \simeq A, \quad y \leftrightarrow\left(\begin{array}{cc}
\sqrt{y} & 0 \\
0 & \sqrt{y}^{-1},
\end{array}\right)
$$

and we set

$$
z(g)=n(g) a(g)
$$

By the map

$$
G \stackrel{\rho}{\rightarrow} \mathcal{H}, \quad \rho(g)=g \cdot i
$$

$z(g)$ may be considered as a point of $\mathcal{H}$. For example, $z(n(x) a(y))$ is identified with $z=x+i y$. For $s \in \mathbf{C}$ and an irreducible representation $\mu$ of $K$, a smooth function $y_{\mu}^{s}$ on $G$ is defined to be

$$
y_{\mu}^{s}(n a k)=\mu(k) y^{s}(a) \text {. }
$$

Note that $y_{\mu}^{s}$ satisfies

$$
y_{\mu}^{s}(g k)=\mu(k) y_{\mu}^{s}(g), \quad k \in K, \quad g \in G .
$$

Every irreducible representation of $K$ is parametrized by an integer (i.e., its weight), we will sometimes identify them. Let $\Sigma$ be the parameter space of irreducible unitary representations of $G$ :

- $\Sigma_{\mathrm{pr}}=\left\{\frac{1}{2}+i \nu \mid \nu \in \mathbf{R}\right\}$, 
- $\Sigma_{\text {comp }}=\{s \in \mathbf{R} \mid 0<s<1\}$,

- For an irreducible representation $\mu$ of $G$, we set

$$
\Sigma_{\text {disc }}^{\mu}=\left\{\frac{k}{2} \mid k \in \mathbf{Z}, k \geq 1, k \equiv \mu(\bmod 2)\right\},
$$

- $\Sigma_{\mu}=\Sigma_{\mathrm{pr}} \cup \Sigma_{\text {comp }} \cup \Sigma_{\text {disc }}^{\mu}$.

Suppose we are given a $(\mathbf{g}, K)$-module whose $K$-type is $\mu$. Then, we may choose its basis from $\left\{y_{\mu}^{s}\right\}_{s \in \Sigma_{\mu}}$. Let $p_{t}^{h}$ be the heat kernel of the positive hyperbolic Laplacian

$$
\Delta=-y^{2}\left(\frac{\partial}{\partial x^{2}}+\frac{\partial}{\partial y^{2}}\right)
$$

By the formula

$$
\Delta y^{s}=s(1-s) y^{s}
$$

we have

$$
\left(e^{-t s(1-s)} y^{s}\right)(z)=\left(e^{-t \Delta} y^{s}\right)(z)=\int_{\mathcal{H}} p_{t}^{h}\left(z, z^{\prime}\right) y^{s}\left(z^{\prime}\right) d z^{\prime}
$$

Here, the integral is taken with respect to the Poincaré metric. Under the isomorphism as $K$-modules,

$$
W^{1.1} \simeq \mathbf{C}(1)^{\oplus d} \oplus \mathbf{C}(-1)^{\oplus d}
$$

a smooth function

$$
Y_{\alpha}^{s}(g)=\left(\alpha_{-1} y_{-1}^{s}(g), \alpha_{1} y_{1}^{s}(g)\right), \quad \alpha=\left(\alpha_{-1}, \alpha_{1}\right), \quad \alpha_{ \pm 1} \in \mathbf{C}^{\oplus d}
$$

may be considered as an element of $\left(C^{\infty}(G) \otimes W^{1.1}\right)^{K}$. Using Example 2.2, Lemma

\section{Lemma 7.1.}

(1) $Y_{\alpha}^{s}$ is an eigenfunction of $\Delta_{H}$ whose eigenvalue is

$$
\lambda=s(1-s)-\frac{1}{4} \text {. }
$$

(2) For $t>0$, we define a smooth function $h_{t}$ on $G \times G$ as

$$
\begin{aligned}
& h_{t}\left(g, g^{\prime}\right)=e^{\frac{t}{4}} p_{t}^{h}\left(z(g), z\left(g^{\prime}\right)\right) \\
& \cdot\left(\begin{array}{cc}
\mu_{-1}(k(g)) \mu_{-1}\left(k\left(g^{\prime}\right)\right)^{-1} 1_{d} & 0 \\
0 & \mu_{1}(k(g)) \mu_{1}\left(k\left(g^{\prime}\right)\right)^{-1} 1_{d}
\end{array}\right) .
\end{aligned}
$$

Then, we have

$$
\int_{G} h_{t}\left(g, g^{\prime}\right) Y_{\alpha}^{s}\left(g^{\prime}\right) d g^{\prime}=e^{-t \lambda} Y_{\alpha}^{s}(g) .
$$


Corollary 7.2. $h_{t}$ is the kernel function of $e^{-t \Delta_{H}}$.

Now, we will compute

$$
2 s \int_{0}^{\infty} e^{-s^{2} t} I(t) d t
$$

where $I(t)$ is the orbital integral of the identity element:

$$
I(t)=\operatorname{vol}(M) \operatorname{Tr} h_{t}\left(1_{2}, 1_{2}\right) .
$$

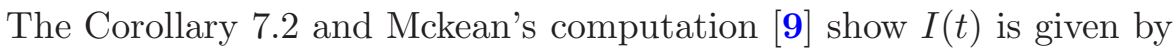

$$
I(t)=\frac{2 d \operatorname{vol}(M)}{(4 \pi t)^{\frac{3}{2}}} \int_{0}^{\infty} \frac{\nu e^{-\frac{\nu^{2}}{4 t}}}{\sinh \frac{\nu}{2}} d \nu .
$$

Also, he has shown

$$
\int_{0}^{\infty} d t \frac{e^{-\left(\sigma(\sigma-1)+\frac{1}{4}\right) t}}{(4 \pi)^{\frac{1}{2}} t^{\frac{3}{2}}} \int_{0}^{\infty} \frac{\nu e^{-\frac{\nu^{2}}{4 t}}}{\sinh \frac{\nu}{2}} d \nu=\sum_{n=0}^{\infty} \frac{1}{\sigma+n}, \quad \sigma \in \mathbf{C} .
$$

Now, by the change of variables

$$
s+\frac{1}{2}=\sigma,
$$

and by the generalized Gauss-Bonnet formula [i]

$$
\operatorname{vol}(M)=-2 \pi \chi(M),
$$

we have

Theorem 7.3.

$$
2 s \int_{0}^{\infty} e^{-s^{2} t} I(t) d t=-2 s d \chi(M) \sum_{n=0}^{\infty} \frac{1}{s+\frac{1}{2}+n} .
$$

Note that the poles of the integral are located on

$$
\left\{-\frac{1}{2}-n\right\}_{n \in \mathbf{Z}, n \geq 0}
$$

and the residue at $s=-(1 / 2)-n$ is equal to $d(1+2 n) \chi(M)$. In particular, they are all integers.

Next, we will compute the orbital integral over parabolic orbits. Let $\gamma$ be a parabolic element which conjugate to $\left(\begin{array}{cc}1 & m \\ 0 & 1\end{array}\right)$. The orbital integral associated to the conjugacy class of $\gamma$ is defined to be [1.

$$
P_{t}(\gamma)=\frac{1}{2 \pi} \int_{A K} \operatorname{Tr} h_{t}(g, \gamma g) d a d k
$$


Since $\gamma$ is parabolic, $k(\gamma g)=k(g)$. Therefore, we have

$$
h_{t}(g, \gamma g)=e^{\frac{t}{4}} p_{t}^{h}(z(g), z(\gamma g)) 1_{2 d},
$$

and in particular,

$$
\operatorname{Tr} h_{t}(g, \gamma g)=2 d e^{\frac{t}{4}} p_{t}^{h}(z(g), z(\gamma g)) .
$$

It is known that $p_{t}^{h}$ can be expressed by a smooth function $k_{t}$ on $\mathbf{R}_{>0}$ [8;i]:

$$
p_{t}^{h}\left(z, z^{\prime}\right)=k_{t}\left(\frac{\left|z-z^{\prime}\right|^{2}}{y y^{\prime}}\right), \quad z=x+i y, \quad z^{\prime}=x^{\prime}+i y^{\prime} .
$$

So, we have

$$
P_{t}(\gamma)=2 d e^{\frac{t}{4}} \int_{0}^{\infty} k_{t}\left(\frac{m^{2}}{y^{2}}\right) \frac{d y}{y^{2}}
$$

For $Y>0$, we set

$$
P_{t}(\gamma)_{Y}=2 d e^{\frac{t}{4}} \int_{0}^{Y} k_{t}\left(\frac{m^{2}}{y^{2}}\right) \frac{d y}{y^{2}}
$$

The formula

$$
\int_{-\infty}^{\infty} e^{-t \nu^{2}} d \nu=\sqrt{\frac{\pi}{t}}
$$

and Kubota's computation ['] is] imply the following proposition.

Proposition 7.4. Let $h$ be the number of cusps for $\Gamma$. Then, we obtain

$$
\begin{aligned}
& \sum_{\gamma} P_{t}(\gamma)_{Y} \\
& \quad=d h\left(\frac{\log Y}{\sqrt{\pi t}}-\frac{1}{\pi} \int_{-\infty}^{\infty} e^{-t \nu^{2}} \frac{\Gamma^{\prime}(1+i \nu)}{\Gamma(1+i \nu)} d \nu-\frac{\log 2}{\sqrt{\pi t}}+\frac{e^{\frac{t}{4}}}{2}\right)+o_{Y}(1),
\end{aligned}
$$

where $\gamma$ runs through conjugacy classes of parabolic elements and $\lim _{Y \rightarrow \infty} o_{Y}(1)=0$.

Next, we will compute a contribution from the continuous spectra. Each of $L^{2}(M, \mathbf{C})$ and $L^{2}\left(M, \mathcal{W}^{1.1}\right)$ is a $G$-module by the right regular representation. For an irreducible representation $\pi$ of $G$, the isotypical component of $L^{2}(M, \mathbf{C})$ (resp. $L^{2}\left(M, \mathcal{W}^{1.1}\right)$ ) will be denoted by $L^{2}(M, \mathbf{C})(\pi)$ (resp. $\left.L^{2}\left(M, \mathcal{W}^{1.1}\right)(\pi)\right)$. It is known $L^{2}\left(M, \mathcal{W}^{1.1}\right)_{\text {cont }}$ is the direct integral over principal series $\left.\pi_{\nu}=\pi_{\nu, 1}[1] 2\right]$ :

$$
L^{2}\left(M, \mathcal{W}^{1.1}\right)_{\mathrm{cont}}=\int_{\mathbf{R}}^{\oplus} L^{2}\left(M, \mathcal{W}^{1.1}\right)\left(\pi_{\nu}\right) d \nu .
$$


Let $k_{t}^{\nu}$ be the kernel function of $\left.e^{-t \Delta}\right|_{L^{2}(M, \mathbf{C})\left(\pi_{\nu}\right)}$. Since we know

$$
\left.e^{-t \Delta}\right|_{L^{2}(M, \mathbf{C})\left(\pi_{\nu}\right)}=e^{-t\left(\frac{1}{4}+\nu^{2}\right)} \mathrm{Id},
$$

by (8), the equation (6) implies the kernel function of $\left.e^{-t \Delta_{H}}\right|_{L^{2}\left(M, \mathcal{W}^{1.1}\right)\left(\pi_{\nu}\right)}$ is equal to $e^{\frac{t}{4}} k_{t}^{\nu}\left(z, z^{\prime}\right) 1_{2 d}$. Hence, we get

$$
\operatorname{Tr}\left[\left.e^{-t \Delta_{H}}\right|_{L^{2}\left(M, \mathcal{W}^{1.1}\right)_{\mathrm{cont}}}\right]=2 d e^{\frac{t}{4}} \int_{-\infty}^{\infty} d \nu \int_{D_{\Gamma}} k_{t}^{\nu}(z, z) d z,
$$

where $D_{\Gamma}$ is the fundamental domain for $\Gamma$. Let $\left\{\kappa_{1}, \ldots, \kappa_{h}\right\}$ be the cusps for $\Gamma$ and let $E_{j}(z, s)$ be the Eisenstein series corresponding to $\kappa_{j}$. Since it is known that

$$
k_{t}^{\nu}\left(z, z^{\prime}\right)=\frac{e^{-\left(\frac{1}{4}+\nu^{2}\right) t}}{4 \pi} \sum_{j=1}^{h} E_{j}\left(z, \frac{1}{2}+i \nu\right) \overline{E_{j}\left(z^{\prime}, \frac{1}{2}+i \nu\right)},
$$

we have

$$
\begin{aligned}
\operatorname{Tr} & {\left[\left.e^{-t \Delta_{H}}\right|_{L^{2}\left(M, \mathcal{W}^{1.1}\right)_{\mathrm{cont}}}\right] } \\
& =\frac{d}{2 \pi} \sum_{j=1}^{h} \int_{D_{\Gamma}} d z \int_{-\infty}^{\infty} e^{-t \nu^{2}} E_{j}\left(z, \frac{1}{2}+i \nu\right) \overline{E_{j}\left(z, \frac{1}{2}+i \nu\right)} d \nu .
\end{aligned}
$$

For $Y>0$, we set

$$
D_{\Gamma}(Y)=\left\{z \in D_{\Gamma} \mid \operatorname{Im} z \leq Y\right\}
$$

The following identity is proved in [8] Appendix:

$$
\begin{aligned}
& \frac{1}{4 \pi} \sum_{j=1}^{h} \int_{D_{\Gamma}(Y)} d z \int_{-\infty}^{\infty} e^{-t\left(\frac{1}{4}+\nu^{2}\right)} E_{j}\left(z, \frac{1}{2}+i \nu\right) \overline{E_{j}\left(z, \frac{1}{2}+i \nu\right)} d \nu \\
& \quad=\frac{h e^{\frac{-t}{4}}}{2 \sqrt{\pi t}} \log Y-\frac{1}{4 \pi} \int_{-\infty}^{\infty} e^{-t\left(\frac{1}{4}+\nu^{2}\right)} \frac{\Psi^{\prime}\left(\frac{1}{2}+i \nu\right)}{\Psi\left(\frac{1}{2}+i \nu\right)} d \nu+\frac{1}{4} \operatorname{Tr} \Phi\left(\frac{1}{2}\right)+o_{Y}(1) .
\end{aligned}
$$

We will explain the terminologies.

The constant term $a_{i j, 0}=a_{i j, 0}(y, s)$ of the Fourier expansion of $E_{i}(z, s)$ at $\kappa_{j}$ can be written as

$$
a_{i j, 0}=\delta_{i j} y^{s}+\varphi_{i j, 0}(s) y^{1-s}
$$

where $\varphi_{i j, 0}(s)$ is a meromorphic function on $\mathbf{C}$. We set

$$
\varphi_{i j}(s)=\frac{\sqrt{\pi} \Gamma\left(s-\frac{1}{2}\right)}{\Gamma(s)} \varphi_{i j, 0}(s) .
$$




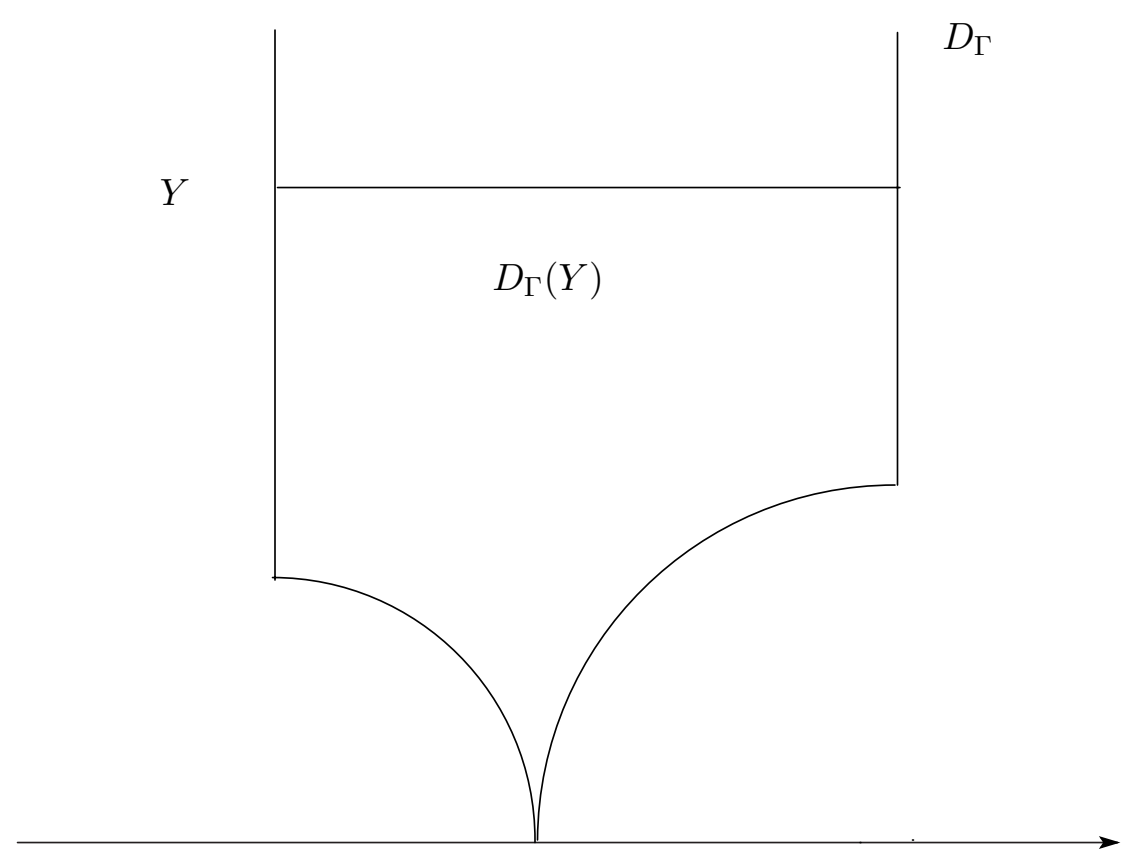

Figure 1.

and the matrix valued function $\Phi(s)$ is defined as

$$
\Phi(s)=\left(\varphi_{i j}(s)\right)_{1 \leq i, j \leq h} .
$$

It is known that $\Phi(s)$ satisfies

$$
\Phi(s) \Phi(1-s)=1_{h}
$$

and that $\Phi(s)$ is a unitary matrix for $s \in(1 / 2)+i \mathbf{R}[8]$ [ In particular, $\Phi(1 / 2)$ is conjugate to

$$
\left(\begin{array}{cccc}
\epsilon_{1} & 0 & \ldots & 0 \\
0 & \epsilon_{2} & \ldots & 0 \\
\vdots & \vdots & \ddots & 0 \\
0 & \ldots & 0 & \epsilon_{h}
\end{array}\right), \quad \epsilon_{i} \in\{ \pm 1\}
$$

Let $\nu_{1}\left(\right.$ resp. $\left.\nu_{-1}\right)$ be the cardinal of $\left\{\epsilon_{i} \mid \epsilon_{i}=1\right\}$ (resp. $\left.\left\{\epsilon_{i} \mid \epsilon_{i}=-1\right\}\right)$. Then, we have

$$
\frac{1}{2}\left(h-\operatorname{Tr} \Phi\left(\frac{1}{2}\right)\right)=\nu_{-1} .
$$

$\Psi(s)$ is defined to be the determinant of $\Phi(s)$. It is known that $\Psi(1 / 2)+$ $\nu)(\nu \in \mathbf{R})$ satisfies the following properties [i]ㄱㅁ. 


\section{Fact 7.5.}

(1) $\Psi\left(\frac{1}{2}+\nu\right) \Psi\left(\frac{1}{2}-\nu\right)=1$.

(2) $\Psi\left(\frac{1}{2}+\nu\right)$ is a ratio of entire functions $p$ and $q$ of finite order,

$$
\Psi\left(\frac{1}{2}+\nu\right)=\frac{p(\nu)}{q(\nu)}
$$

(3) We have

$$
\frac{\left|\Psi^{\prime}\left(\frac{1}{2}+\nu\right)\right|}{\left|\Psi\left(\frac{1}{2}+\nu\right)\right|} \leq \text { Const } \cdot(\log |\nu|)^{2}
$$

for $|\nu| \geq 2$.

(4) There exists an entire function $r(\nu)$ such that

$$
p(\nu)=q(-\nu) e^{r(\nu)} \text {. }
$$

(5) $\frac{\Psi^{\prime}\left(\frac{1}{2}+\nu\right)}{\Psi\left(\frac{1}{2}+\nu\right)}$ is regular on the imaginary axis and its poles are located on $\left\{ \pm q_{k}\right\}_{k}\left(\operatorname{Re} q_{k}>0\right)$. Moreover, their residues satisfy

$$
b_{k}=\operatorname{Res}_{\nu=q_{k}} \frac{\Psi^{\prime}\left(\frac{1}{2}+\nu\right)}{\Psi\left(\frac{1}{2}+\nu\right)}=-\operatorname{Res}_{\nu=-q_{k}} \frac{\Psi^{\prime}\left(\frac{1}{2}+\nu\right)}{\Psi\left(\frac{1}{2}+\nu\right)} .
$$

Now, $P(t)$ is defined to be

$$
\begin{aligned}
P(t)=\lim _{Y \rightarrow \infty}\left\{\sum_{\gamma} P_{t}(\gamma)_{Y}-\frac{d}{2 \pi} \sum_{j=1}^{h} \int_{D_{\Gamma}(Y)} d z\right. \\
\left.\cdot \int_{-\infty}^{\infty} e^{-t \nu^{2}} E_{j}\left(z, \frac{1}{2}+i \nu\right) \overline{E_{j}\left(z, \frac{1}{2}+i \nu\right)} d \nu\right\},
\end{aligned}
$$

which may be considered as

$$
\sum_{\gamma} P_{t}(\gamma)-\operatorname{Tr}\left[\left.e^{-t \Delta_{H}}\right|_{L^{2}\left(M, \mathcal{W}^{1.1}\right)_{\mathrm{cont}}}\right]
$$

Putting altogether, we have

\section{Theorem 7.6.}

$$
\begin{aligned}
P(t)=d\left(\frac{1}{2 \pi} \int_{-\infty}^{\infty} e^{-t \nu^{2}} \frac{\Psi^{\prime}\left(\frac{1}{2}+i \nu\right)}{\Psi\left(\frac{1}{2}+i \nu\right)} d \nu\right. \\
\left.\quad-\frac{h}{\pi} \int_{-\infty}^{\infty} e^{-t \nu^{2}} \frac{\Gamma^{\prime}(1+i \nu)}{\Gamma(1+i \nu)} d \nu-\frac{h}{\sqrt{\pi t}} \log 2+\nu_{-1} e^{\frac{t}{4}}\right)
\end{aligned}
$$

Next, we will compute

$$
2 s \int_{0}^{\infty} e^{-s^{2} t} P(t) d t
$$


for suitable $s \in \mathbf{C}$. First of all, the integral

$$
2 s \int_{0}^{\infty} e^{-\left(s^{2}-\frac{1}{4}\right) t} d t
$$

absolutely converges for $\operatorname{Re} s^{2}>\frac{1}{4}$ and can be meromorphically continued throughout the whole plane as

$$
\frac{1}{s+\frac{1}{2}}+\frac{1}{s-\frac{1}{2}}
$$

On the other hand, since we have

$$
s \int_{0}^{\infty} \frac{e^{-s^{2} t}}{\sqrt{t}} d t=\sqrt{\pi}
$$

for $s>0$, LHS may be continued over the whole plane as the constant function $\sqrt{\pi}$.

Proposition 7.7. For $s \in \mathbf{C}$ with $-\frac{\pi}{4}<\arg s<\frac{\pi}{4}$, we have

$$
\frac{s}{\pi} \int_{0}^{\infty} d t e^{-s^{2} t} \int_{-\infty}^{\infty} e^{-t \nu^{2}} \frac{\Gamma^{\prime}(1+i \nu)}{\Gamma(1+i \nu)} d \nu=\frac{\Gamma^{\prime}(1+s)}{\Gamma(1+s)}
$$

$$
\begin{aligned}
\frac{s}{\pi} \int_{0}^{\infty} d t e^{-s^{2} t} \int_{-\infty}^{\infty} e^{-t \nu^{2}} \frac{\Psi^{\prime}\left(\frac{1}{2}+i \nu\right)}{\Psi\left(\frac{1}{2}+i \nu\right)} d \nu \\
=\frac{\Psi^{\prime}\left(\frac{1}{2}-s\right)}{\Psi\left(\frac{1}{2}-s\right)}-\sum_{k} b_{k}\left(\frac{1}{s+q_{k}}+\frac{1}{s-q_{k}}\right)
\end{aligned}
$$

Proof. $\frac{\Gamma^{\prime}(z)}{\Gamma(z)}$ has poles on non-positive integers and we obtain an estimate

$$
\frac{\left|\Gamma^{\prime}(1+i \nu)\right|}{|\Gamma(1+i \nu)|} \leq \text { Const } \cdot \log |\nu|, \quad|\nu| \geq 2 .
$$

For $s \in \mathbf{C}$ with $-\frac{\pi}{4}<\arg s<\frac{\pi}{4}$, a simple computation shows

$$
s \int_{0}^{\infty} d t e^{-s^{2} t} \int_{-\infty}^{\infty} e^{-t \nu^{2}} \frac{\Gamma^{\prime}(1+i \nu)}{\Gamma(1+i \nu)} d \nu=s \int_{-\infty}^{\infty} \frac{1}{\nu^{2}+s^{2}} \frac{\Gamma^{\prime}(1+i \nu)}{\Gamma(1+i \nu)} d \nu .
$$

Note that the poles of $\frac{\Gamma^{\prime}(1+i \nu)}{\Gamma(1+i \nu)}$ are $\nu=i, 2 i, 3 i, \ldots$ Using the estimate above, one may apply the residue theorem for the contour in Figure Then, one will find

$$
\int_{-\infty}^{\infty} \frac{s}{\nu^{2}+s^{2}} \frac{\Gamma^{\prime}(1+i \nu)}{\Gamma(1+i \nu)} d \nu=\pi \frac{\Gamma^{\prime}(1+s)}{\Gamma(1+s)} .
$$




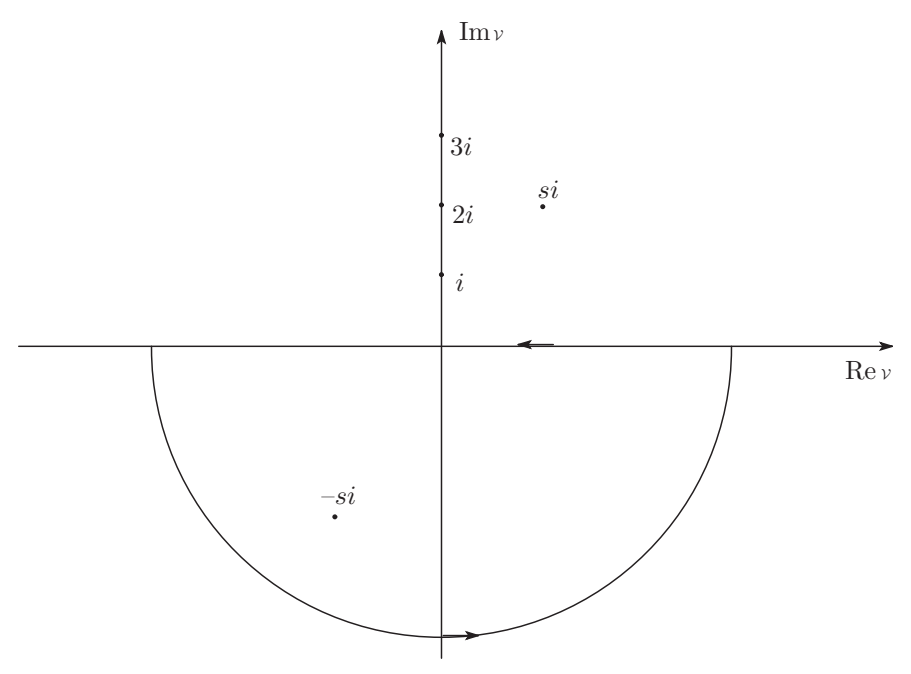

Figure 2.

Thus, (1) is proved. Taking account of Fact 7.5 (3) and (5), (2) will be proved by the same way.

q.e.d.

We set

$$
F(s)=\frac{\Psi^{\prime}\left(\frac{1}{2}-s\right)}{\Psi\left(\frac{1}{2}-s\right)}-\sum_{k} b_{k}\left(\frac{1}{s+q_{k}}+\frac{1}{s-q_{k}}\right) .
$$

Then, Fact 7.5(5) implies the poles of $F(s)$ are located on $\left\{ \pm q_{k}\right\}_{k}$ and each of them is simple. Moreover, we have

$$
\operatorname{Res}_{s=q_{k}} F(s)=0, \quad \operatorname{Res}_{s=-q_{k}} F(s)=-2 b_{k} .
$$

Hence, $F(s)$ is regular on $\{s \in \mathbf{C} \mid \operatorname{Re} s \geq 0\}$. Combining altogether, we obtain the following theorem. 
Theorem 7.8. The integral $2 s \int_{0}^{\infty} e^{-s^{2} t} P(t) d t$ can be meromorphically continued to the whole plane as

$$
\begin{aligned}
2 s \int_{0}^{\infty} e^{-s^{2} t} P(t) d t=d & \frac{\Psi^{\prime}\left(\frac{1}{2}-s\right)}{\Psi\left(\frac{1}{2}-s\right)}-\sum_{k} b_{k}\left(\frac{1}{s+q_{k}}+\frac{1}{s-q_{k}}\right) \\
& +\nu_{-1}\left(\frac{1}{s+\frac{1}{2}}+\frac{1}{s-\frac{1}{2}}\right) \\
& \left.-2 h \frac{\Gamma^{\prime}(1+s)}{\Gamma(1+s)}-2 h \log 2\right) .
\end{aligned}
$$

It has only simple poles whose residues are integers. Moreover, it is regular at $s=0$.

Now, the analytic continuation of $Z_{S}(s)$ is proved.

Theorem 7.9. $Z_{S}(s)$ can be meromorphically continued throughout the s-plane as

$$
\begin{aligned}
Z_{S}(s)= & \frac{2 b}{s}+\sum_{n=b+1}^{\infty}\left(\frac{1}{s+\sqrt{\lambda_{n}} i}+\frac{1}{s-\sqrt{\lambda_{n}} i}\right) \\
& +2 s d \chi(M) \sum_{n=0}^{\infty} \frac{1}{s+\frac{1}{2}+n}-d\left(\frac{\Psi^{\prime}\left(\frac{1}{2}-s\right)}{\Psi\left(\frac{1}{2}-s\right)}\right. \\
& \left.-\sum_{k} b_{k}\left(\frac{1}{s+q_{k}}+\frac{1}{s-q_{k}}\right)+\nu_{-1}\left(\frac{1}{s+\frac{1}{2}}+\frac{1}{s-\frac{1}{2}}\right)\right) \\
& +2 d h\left(\frac{\Gamma^{\prime}(1+s)}{\Gamma(1+s)}+\log 2\right) .
\end{aligned}
$$

RHS has only simple poles and every residue is an integer. Its poles are located in $\{s \in \mathbf{C} \mid \operatorname{Re} s \leq 0\}$ except for $s=1 / 2$. Moreover, we have

$$
\operatorname{Res}_{s=0} Z_{S}(s)=2 b \text {. }
$$

Since $Z_{S}(s)$ is the logarithmic derivative of $\zeta_{S}(s)$, Theorem

Theorem 7.10. $\zeta_{S}(s)$ can be meromorphically continued to the whole plane. Also, it satisfies

$$
\operatorname{ord}_{s=0} \zeta_{S}(s)=2 b \text {. }
$$

By definition, the logarithmic derivative of $\zeta_{R}(s)$ is

$$
\frac{\zeta_{R}^{\prime}(s)}{\zeta_{R}(s)}=Z_{S}\left(s-\frac{1}{2}\right)-Z_{S}\left(s+\frac{1}{2}\right) .
$$


and Theorem

$$
\operatorname{Res}_{s=\frac{1}{2}} \frac{\zeta_{R}^{\prime}(s)}{\zeta_{R}(s)}=2 b .
$$

Theorem 7.11. The Ruelle zeta function $\zeta_{R}(s)$ can be meromorphically continued throughout the s-plane and it has a zero at $s=1 / 2$ of order $2 b$.

Taking account of Proposition $\overline{6} \cdot \overline{6}$, this implies the logarithmic derivative of the "Euler product"

$$
\prod_{\gamma_{0} \in \Gamma_{\mathrm{pr}, \text { conj }}^{*}} \operatorname{det}\left[1_{2 d}-\rho\left(\gamma_{0}\right) e^{-s l\left(\gamma_{0}\right)}\right]
$$

can be meromophically continued throughout the whole plane and its residue at $s=1 / 2$ is equal to $b$.

\section{A geometric application}

Let $X$ be a smooth quasi-projective variety with a fibration

$$
X \stackrel{f}{\longrightarrow} S
$$

as in the introduction. We will use the notation of the Condition 1.1 .

Let $\mathcal{V}_{\mathbf{C}}$ be the flat vector bundle over $S_{0}$ which associates to the locally constant sheaf $R^{1} f_{*} \mathbf{C} . V_{\mathbf{C}}=V \otimes_{\mathbf{R}} \mathbf{C}$ has a direct decomposition

$$
V_{\mathbf{C}}=(\mathbf{C}(1) \oplus \mathbf{C}(-1))^{\oplus d}
$$

as a $K$-module and we may form a homogeneous vector bundle on $\mathcal{H}$

$$
\tilde{\mathcal{V}}_{\mathbf{C}}=G \times_{K} V_{\mathbf{C}} \text {. }
$$

Then $\mathcal{V}_{\mathbf{C}}$ is the descent of $\tilde{\mathcal{V}}_{\mathbf{C}}$ using the monodromy representation. Let $\mathcal{W}^{p \cdot q}$ be the same as in Section rational Mordell-Weil group $X(S) \otimes \mathbf{Q}$ is of finite dimension and by the cycle map it may be considered as a subspace of $H^{1}\left(S_{0}, R^{1} f_{*} \mathbf{Q}\right)$. Note that the Hodge-Lefshetz theorem implies

$$
X(S) \otimes \mathbf{Q}=H^{1}\left(S_{0}, R^{1} f_{*} \mathbf{Q}\right) \cap \operatorname{Ker}\left[\Delta_{H} \mid L^{2}\left(S_{0}, \mathcal{W}^{1.1}\right)\right] .
$$

Moreover, we have

$H^{1}\left(S_{0}, R^{1} f_{*} \mathbf{Q}\right) \cap \operatorname{Ker}\left[\Delta_{H} \mid L^{2}\left(S_{0}, \mathcal{W}^{2.0} \oplus \mathcal{W}^{0.2}\right)\right] \subset H^{2.0}(X) \oplus H^{0.2}(X)$

by the compatibility of the Hodge decomposition and the Leray spectral

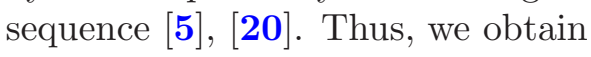

Theorem 8.1. The rank of the Mordell-Weil group is less than or equal to the dimension of $\operatorname{Ker}\left[\Delta_{H} \mid L^{2}\left(S_{0}, \mathcal{W}^{1.1}\right)\right]$. Moreover, if $H^{2}(X$, $\left.\mathcal{O}_{X}\right)=0$, they are equal. 
We define the Selberg zeta function $\zeta_{S, f}(s)$ (resp. Ruelle zeta function $\left.\zeta_{R, f}(s)\right)$ of the fibration $f$ to be one associated to $\rho_{X}$. Theorems 10, 10 and a solution of geometric analogue of the BSD conjecture.

Theorem 8.2. We have

$$
2 \operatorname{dim}_{\mathbf{Q}} X(S) \otimes \mathbf{Q} \leq \operatorname{ord}_{s=0} \zeta_{S, f}(s)=\operatorname{ord}_{s=\frac{1}{2}} \zeta_{R, f}(s) .
$$

Moreover, if $H^{2}\left(X, \mathcal{O}_{X}\right)=0$, we have the equality in the above formula.

The Proposition 6.6 and the above theorem imply the following:

Theorem 8.3 (A geometric analogue of the BSD conjecture over $\mathbf{C}$ ). The Euler product

$$
L_{X \backslash S}(s)=\prod_{\gamma_{0} \in \Gamma_{\mathrm{pr}, \mathrm{conj}}^{*}} \operatorname{det}\left[1_{2 d}-\rho_{X}\left(\gamma_{0}\right) e^{-s l\left(\gamma_{0}\right)}\right]
$$

has a virtual zero at $s=1 / 2$ whose order is greater than or equal to the rank of the Mordell-Weil group. Moreover, if $H^{2}\left(X, \mathcal{O}_{X}\right)=0$, they are equal.

Let us define the topological Brauer group $B r_{\text {top }}(X)$ of $X$ as

$$
B r_{\text {top }}(X)=H^{2}\left(X, \mathcal{O}_{X}^{*}\right)
$$

where the cohomology is taken with respect to the classical topology.

Proposition 8.4. The topological Brauer group of $X$ is finitely generated if and only if $H^{2}\left(X, \mathcal{O}_{X}\right)$ vanishes.

Proof. The exponential sequence

$$
0 \rightarrow \mathbf{Z} \rightarrow \mathcal{O}_{X} \rightarrow \mathcal{O}_{X}^{*} \rightarrow 0
$$

implies an exact sequence

$$
H^{2}(X, \mathbf{Z}) \rightarrow H^{2}\left(X, \mathcal{O}_{X}\right) \rightarrow B r_{\text {top }}(X) \rightarrow H^{3}(X, \mathbf{Z})
$$

Since $X$ is quasi-projective, both $H^{2}(X, \mathbf{Z})$ and $H^{3}(X, \mathbf{Z})$ are finitely generated and our assertion is clear. q.e.d.

Thus, we know that the condition $H^{2}\left(X, \mathcal{O}_{X}\right)=0$ corresponds to finiteness of $l$-part of the Brauer group of Artin's theorem. 


\section{References}

[1] D. Barbasch \& H. Moscovici, $L^{2}$-index theorem and the Selberg trace formula, J. Functional Anal. 53 (1983) 151-201, MR 0722507, Zbl 0537.58039.

[2] B.J. Birch \& H.P.F. Swinnerton-Dyer, Notes_on elliptic_curves I, J. reine u. angewandte Math. 212 (1963) 7-25, MR p179168, Zbl 0118.27601.

[3] B.J. Birch and H.P.F. Swinnerton-Dyer, Notes on elliptic curves II, J. reine u. angewandte Math. 218 (1965) 79-108, MR 0146143, Zbl 0147.02506.

[4] D. Bump. Automorphic Forms and Representation, Number 55 in Cambridge Stud. Adv. Math. Cambridge University Press, 1997, MR 1431508, Zbl 0868.11022

[5] D.A. Cox \& S. Zucker, Intersection numbers of sections of elliptic surfaces, Invent. math. 53 (1979) 1-44, MR 0538682, Zbl 0444.14004 .

[6] D. Fried, Analytic torsion and closed geodesics on hyperbolic manifolds, Invent. Math. 84 (1986) 523-540, MR D837526! Zbl 0621.53035.

[7] R. Gangolli \& G. Warner, Zeta functions of Selberg's type for some noncompact quotient of symmetric space of rank one, Nagoya Math. J. 78 (1980) 1-44, MR 0571435

[8] T. Kubota, Elementary_Theory of Eisenstein Series, Kodansha, Tokyo, 1973, MR

[9] H.P. McKean. Selberg's trace formula as applied to a compact Riemann $\underline{n}$ surface, Comm. Pure Appl. Math. 25 (1972) 225-246, MR 0473167, Zbl 0317.30018.

[10] J.J. Millson, Closed geodesics and the $\eta$-invariant, Ann. Math. 108 (1978) 1-39, MR $0501204, \mathrm{Zbl}, 0399.58020$.

[11] D.R. Morrison, The Clemens-Schmid exact sequence and applications, in 'Topics in Transcendental Algebraic Geometry' (P. Griffith, ed.), Ann. Math. Stud., 106, 101-119, Princeton University Press, 1984, MR 0756848, Zbl 0576.32034 .

[12] W. Müller, Manifolds with Cusps of Rank One, 1244, Springer-Verlag, 1987, MR "0891654, Zbl '0632.58001.

[13] A. Selberg, Harmonic analysis and discontinuous groups in weakly symmetric spaces with application to Dirichlet series, J. Indian Math. Soc. 20 (1956) 4787, MR 0088511 , Zbl 0072.08201 .

[14] S. Shokranian, The Selberg-Authur Trace Formula, Lecture Notes in Math. 1503, Springer-Verlag, 1992, MR 1176101, Zbl 0769.11026.

[15] J. Tate. Algebraic cycles and poles of zeta functions, in 'Proc. of the Purdue Conference on Arithmetic Algebraic Geometry', Harper and Row, 1964, MR 0225778, Zbl, 0213.22804 .

[16] J. Tate. On the conjecture of Birch and Swinnerton-Dyer and a geometric analog, in 'Seminaire Bourbaki', 306, 1-26, 1966, MR 1610977, Zbl 199.55604.

[17] G. van der Geer, Hilbert Modular Surfaces,__ series of Modern_ Surveys in Mathematics, 16, Springer-Verlag, 1988, MR 0930101, Zbl 0634.14022.

[18] N. Wallach. An asymptotic formula of Gelfand and Gangolli for spectrum of $\Gamma \backslash G$, J. Differential Geom. 11 (1976) 91-101, MR 0417340, Zbl 0341.43009.

[19] N. Wallach. On the Selberg trace formula in the case of compact quotient, Bull. AMS 82 (1976) 171-195, MR 0404533, Zbl 0350.22008. 
[20] S. Zucker. Hodge theory with degenerating coefficients, Ann. Math. 109 (1979) 415-476, MR 0534758, Zbl 0446.14002!

DePARTMENT OF MATHEMATICS AND INFORMATICS

FACUlTy of SCIENCE Chiba University 1-33 YAYOI-CHO INAGE-KU CHIBA 263-8522 JAPAN

E-mail address: sugiyama@math.s.chiba-u.ac.jp 